\title{
Magnesium intake and lung cancer risk: A systematic review and meta-analysis
}

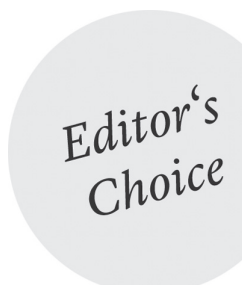

\author{
Nasim Dana ${ }^{1}$, Raheleh Karimi², Marjan Mansourian², Shaghayegh Haghjooy Javanmard ${ }^{1}$, \\ Ismail Laher ${ }^{1,3}$, and Golnaz Vaseghi ${ }^{4}$ (I)

\begin{abstract}
Applied Physiology Research Center, Cardiovascular Research Institute, Isfahan University of Medical sciences, Isfahan, Iran
Isfahan, Iran
\end{abstract} \\ 2 Epidemiology and Biostatistics Department, School of Health, Isfahan University of Medical Sciences, Isfahan, Iran \\ Department of Anesthesiology, Pharmacology and Therapeutics, University of British Columbia, Vancouver, BC, Canada \\ 4 Isfahan Cardiovascular Research Center, Cardiovascular Research Institute, Isfahan University of Medical Sciences,
}

\begin{abstract}
Magnesium may reduce the risk of lung cancer by affecting cell proliferation, inflammation and by preserving lung function; however, the results of epidemiological studies on the potential benefits of magnesium in lung pathology are inconclusive. We conducted this meta-analysis to investigate the association between magnesium intake and the risk of lung cancer. A total of 5 studies were extracted from PubMed, SCOPUS, and the Cochrane Review (to May 2018). These studies involved 58,5821 participants with 8,977 lung cancer cases. The pooled relative risk (RR) indicated a significant association between lung cancer incidence and magnesium intake $(\mathrm{RR}=0.88,95 \% \mathrm{Cl}=0.79$ to $0.98 ; p=0.018)$. To investigate the cause of heterogeneity of these studies $\left(I^{2}=75.8 \%, p<0.001\right)$, we performed a subgroup analysis which was affected by the mean dose of magnesium intake, where doses of magnesium intake lower than $300 \mathrm{mg} / \mathrm{d}$ significantly decreased lung cancer risk ( $R R=0.83,95 \% \mathrm{Cl}=0.70$ to $0.99 ; p=0.034$ ). Increasing magnesium intake doses to over $300 \mathrm{mg} / \mathrm{d}$ did not reduce the incidence of lung cancer $(\mathrm{RR}=0.89,95 \% \mathrm{Cl}=0.78$ to $1.01 ; p=0.076$ ). Our meta-analysis suggests that magnesium intake of less than $300 \mathrm{mg} / \mathrm{d}$ may have protective effects in lung cancer.
\end{abstract}

Keywords: Magnesium, Lung, Cancer, Meta-analysis

\section{Introduction}

Lung cancer is a leading cause of mortality in men and women around the world [1] and is a heterogeneous disease with genomic and phenotypic features [2]. It is estimated that about 228,150 new lung and bronchial cancer cases will be diagnosed in 2019, with the estimated incidence in males (116,440 cases) and females (111,710 cases) representing the second highest causes of all cancer types [3]. Environmental factors such as smoking, gender, asbestosis and pollution are leading causes of lung cancer [4]. Recent epidemiologic evidence suggests that in addition to environmental factors, dietary supplements can also influence the risk of lung cancer [4]. There is limited evidence on the role of dietary mineral intake (e.g. calcium, copper, iron, magnesium, selenium, zinc) in the development of lung cancer [5-9]. Magnesium has metabolic and physiological functions that can influence carcinogenesis [10-12]. It acts as a cofactor in numerous enzymatic reactions and is involved in energy metabolism and the modulation of glucose transport across cell membranes [13]. Magnesium is necessary for genomic stability [12], DNA duplication and repair [14], apoptosis [15] and inhibition of chemical carcinogenesis [16].

There is a complex relationship between magnesium status and tumorigenesis. Magnesium promotes the growth of pre-existing tumors due to the profound changes of magnesium homeostasis in tumor cells, and magnesium insufficiency tends to increase tumor incidence in animals and humans [17]. Low magnesium-induced oxidative stress inhibits endothelial growth and suppresses the formation of new vessels; on the other hand hypomagnesemia can accelerate metastasis as magnesium is a requirement for the function of metastasis-suppressor genes [18] Magnesium can protect against lung cancer by improving genomic stability, regulating cellular proliferation [19], reducing inflammation [20, 21], maintaining pulmonary function [22] and protecting against oxidative stress [23, 24].

The relationship between magnesium intake and the risk of lung cancer is not well documented, although there are 
some reports that magnesium intake affects the incidence of other cancers such as colorectal cancer [25]. We performed a meta-analysis of published observational studies to better quantify the association of magnesium intake and the incidence of lung cancer.

\section{Methods}

\section{Literature search}

A literature search was performed using the Web of Science, PubMed and EMBASE databases through May 2018 and using the search strategy: "lung cancer" or "lung carcinoma" or "lung malignancy" combined with "magnesium intake" or magnesium. No language restrictions were imposed. Two investigators (Vaseghi. G \& Dana. N) reviewed all the retrieved studies independently. Disagreements between the two investigators were resolved by consensus with a third reviewer. The following criteria were used to determine if studies were eligible: (i) case-control or cohort study design, (ii) magnesium intake as a modifier of interest, (iii) lung cancer as the outcome of interest, and (iv) odds ratio (OR) or relative risk (RR) or hazard ratio (HR) estimates with corresponding $95 \%$ confidence intervals (CI).

\section{Data extraction}

Data were extracted from each identified study by using a standardized data-collection form requiring the following data input: the first author's surname, publication year, country of study, source of controls (for case-control studies), length of follow-up (for cohort studies), gender of subjects, sample size, source of magnesium, categorized magnesium intake and corresponding risk estimates that reflected the greatest degree of adjustment, and variables accounting for the statistical model. Magnesium consumption data was extracted from the diets reported in a questionnaire. For measuring the amount of dietary magnesium intake, four of the studies used the food frequency questionnaire (FFQ), and one study has used the Cancer Institute's Health Habits Questionnaire (HHHQ).

\section{Statistical analysis}

An overall analysis of all eligible studies was performed to assess the association between magnesium intake and incidence of lung cancer. We extracted the adjusted effect sizes and $95 \%$ CIs in each study. Of the five studies, three reported hazard ratios (HR), one reported relative risks (RR) and one reported odds ratios (OR) as effect sizes. Since we used the number of events in each study when combining the results, and when the risk was not constant with respect to time, HR was considered to be broadly equivalent to RR [26] and we thus report RR as a pooled effect and created forest plots to determine the multivariate adjusted RR and corresponding 95\% CI. Statistical heterogeneity was evaluated using Cochran $\mathrm{Q}$ and $\mathrm{I}^{2}$ analysis. For the Cochran $\mathrm{Q}$ P values of $<0.10$, and for the $\mathrm{I}^{2}$ analysis, values $>40 \%$, were considered to indicate statistically significant differences in heterogeneity. If the heterogeneity between studies was significant, we then used a random effects model to compute the pooled effect measured with $95 \%$ CIs; in other cases, a fixed effect model was used. A sensitivity analysis was performed by excluding studies individually and by analyzing the homogeneity and effect size for the remaining studies. We used Egger's regression test and the Begg's test to examine publication bias and funnel plots $[27,28]$. A P value $<0.05$ was considered significant, and we used the trim and fill method to correct it [29]. All calculations and graphs were made using Stata version 14.

\section{Results}

\section{Study characteristics}

A flowchart showing the study selection is shown in Figure 1. Our literature search identified five eligible studies published between 2008 and 2017; these studies contained a total of 58,5821 participants and 8,977 lung cancer cases. Characteristics of the studies are shown in Table 1. Two of the five studies were conducted in the United States of America, one in Germany, one in China and the other in the Netherlands.

\section{Magnesium intake}

A summary RRs for magnesium intake and risk of lung cancer is shown in Figure 2. The five eligible studies yielded a pooled RR with $95 \%$ confident interval was not statistically significant $(\mathrm{RR}=0.79,95 \% \mathrm{CI}=0.52$ to $1.19 ; p=0.254)$. The results of all studies were adjusted by sex, age, physical activity, body mass index (BMI), smoking categories, alcohol intake, dietary supplementation and family history of cancer. Based on the adjusted results, magnesium consumption for the highest compared with the lowest doses did not significantly reduce the risk of lung cancer. The effect size was pooled by using a random-effects model as significant heterogeneity was identified $\left(\mathrm{I}^{2}=89.3 \%, \mathrm{p}<\right.$ $0.001)$. This heterogeneity was likely related to a study that used high doses of magnesium ( $>448 \mathrm{mg} / \mathrm{d}$ ) compared with other studies [6]. The heterogeneity between studies 


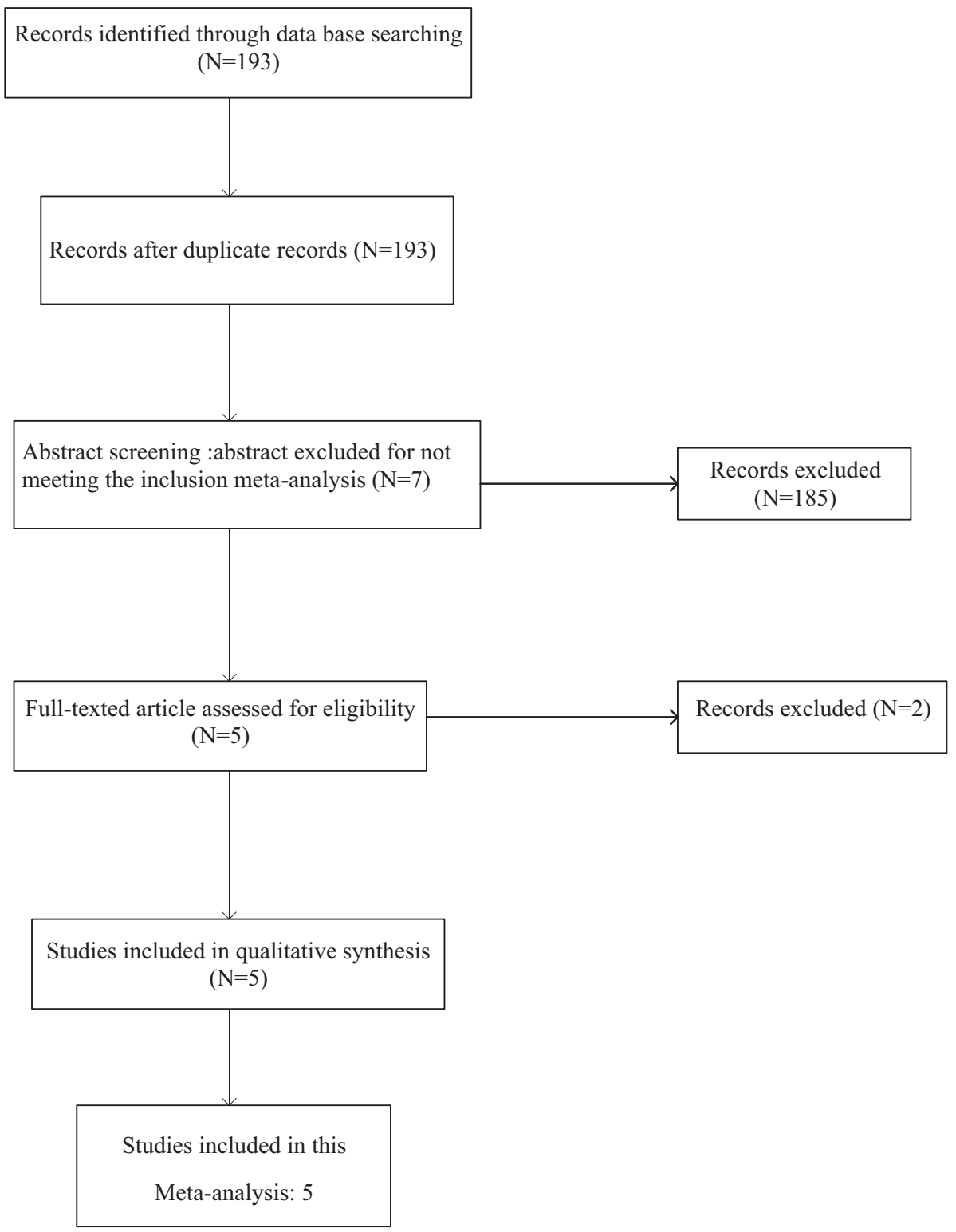

Figure 1. Flow chart showing the process of study selection.

decreased by removing this study from the analysis $\left(\mathrm{I}^{2}=\right.$ $56.6 \%)$.

Statistical evidence of publication bias was obtained using Egger's linear regression tests. Because of the significant $p$-value ( $\mathrm{p}=0.005)$, and from the data in the funnel plot (Figure 2), we used a "trim and fill" method to quantify the publication bias and the modification of the final results from the meta-analysis. The results obtained by this method did not differ from the results of the meta-analysis, indicating good reliability. We performed a sensitivity analysis to determine the impact of each study on the final results. The range of RR estimates was $0.78-0.97$, indicating that the final results were less sensitive when entering the results of each study, and that the meta-analysis model had a higher degree of stability.
In addition, two of studies examined the effect of magnesium consumption on lung cancer separately in men $[5,6]$; by combining the results of these two papers, the pooled RR with $95 \%$ confident interval was not statistically significant $(\mathrm{RR}=0.92,95 \% \mathrm{CI}=0.77$ to $1.11 ; p=0.382$. The effect size was pooled by using the random-effects model as significant heterogeneity was identified $\left(\mathrm{I}^{2}=97.1 \%, \mathrm{p}<0.001\right)$ (Not shown).

\section{Subgroup analysis}

We performed a subgroup analysis to investigate heterogeneity of the studies due the significant effects of mean doses of magnesium intake $(<300(\mathrm{mg} / \mathrm{d})$ vs $>300(\mathrm{mg} / \mathrm{d}))$. 


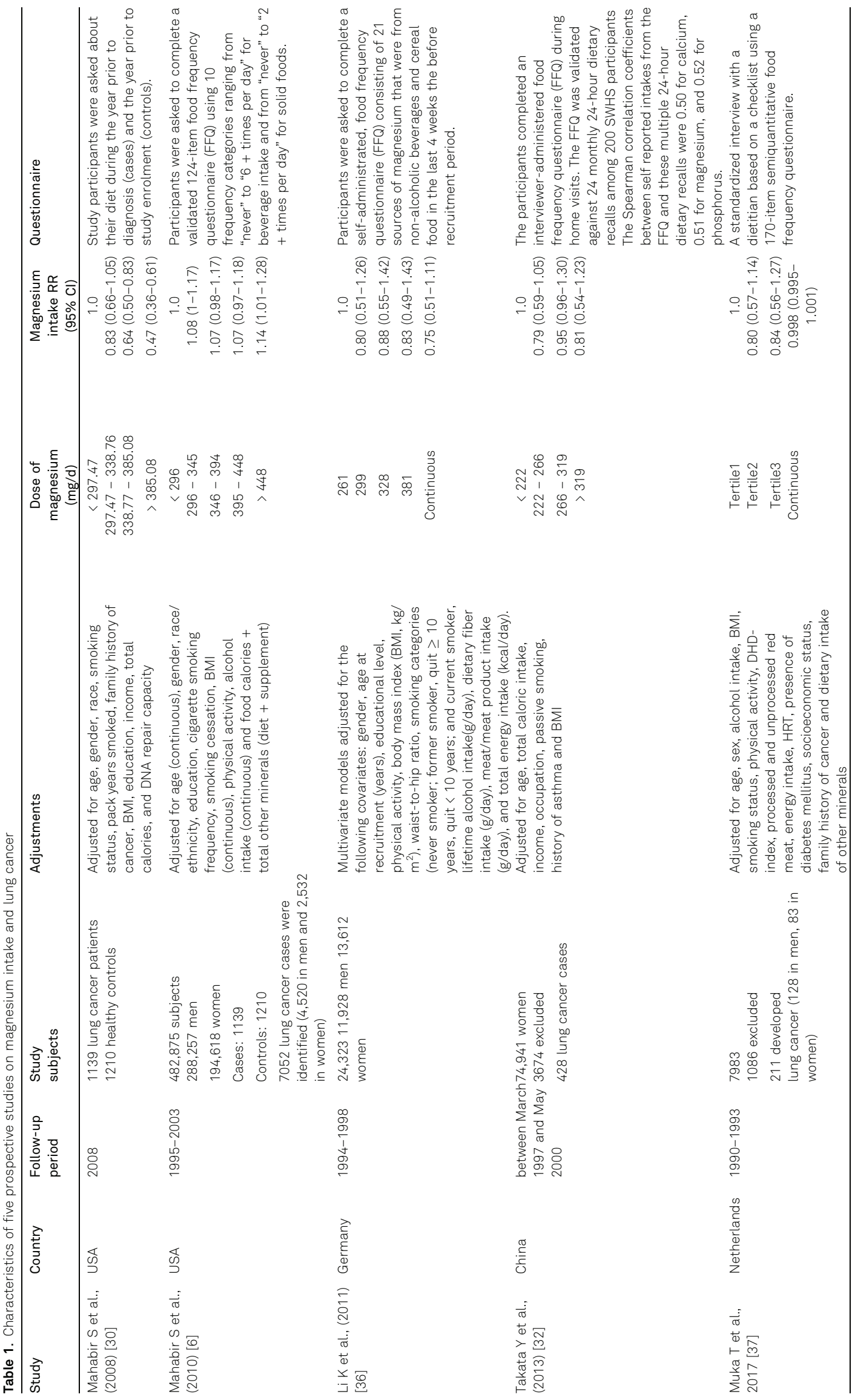




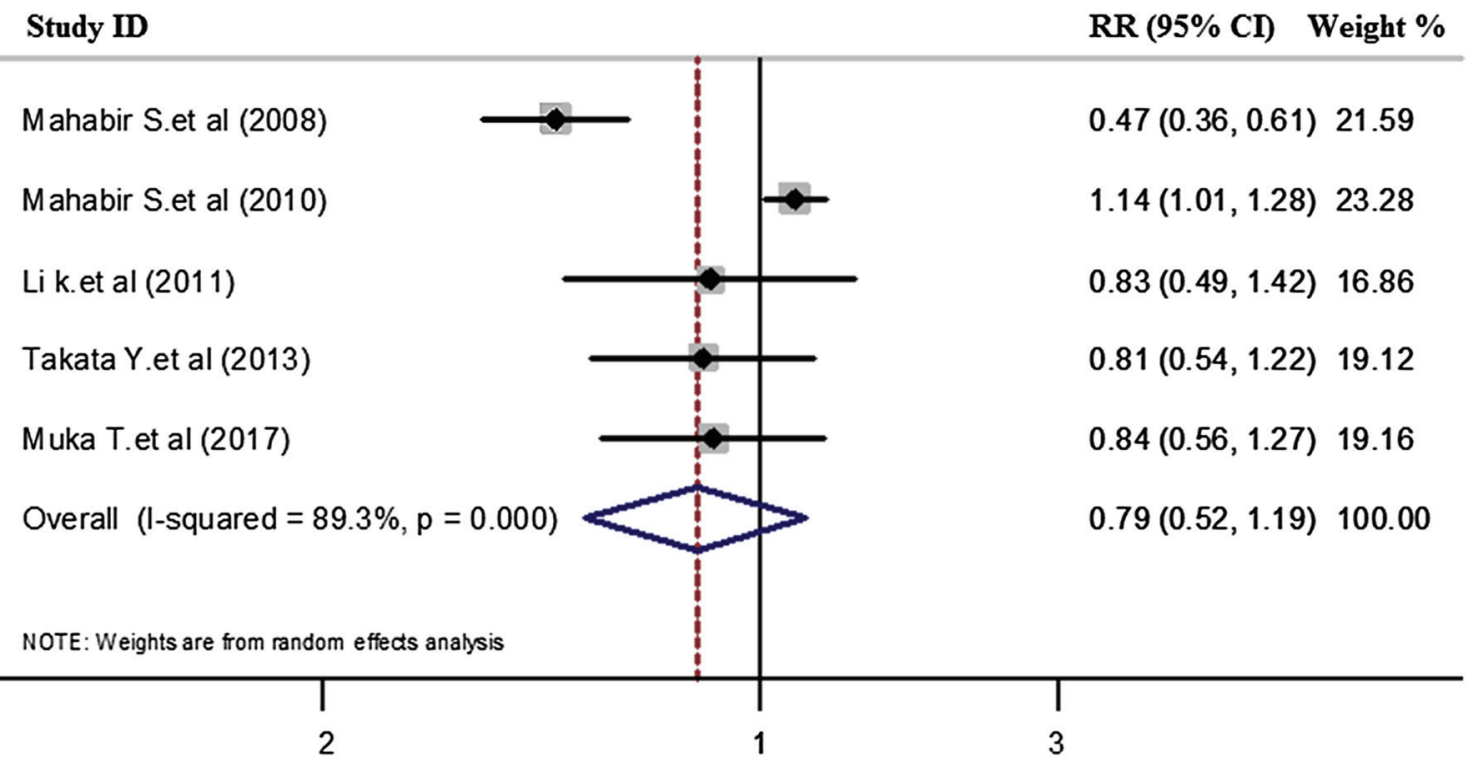

Figure 2. The summary Relative Risk of magnesium intake on risk of lung cancer for the highest compared with the lowest consumption.

Magnesium intake below 300 (mg/d) was associated with a significant decrease in lung cancer risk $(\mathrm{RR}=0.81,95 \% \mathrm{CI}=$ 0.70 to $0.94 ; p=0.006$ ). There was no significant association between magnesium intake of more than $300(\mathrm{mg} / \mathrm{d})$ and lung cancer $(\mathrm{RR}=0.79,95 \% \mathrm{CI}=0.52$ to $1.19 ; p=$ 0.254 ), as shown in Figure 3.

\section{Quality Assessment}

We used the Newcastle Ottawa Scale (NOS) to assess the quality of observational studies as all of the studies used either case-control or cohort protocols. The NOS measurement tool evaluates studies in three categories: selection,

\begin{tabular}{|c|c|}
\hline Study ID & RR $(95 \%$ CI) Weight \% \\
\hline \multicolumn{2}{|l|}{$<300 \mathrm{mg} / \mathrm{d}$} \\
\hline Mahabir S.et al (2008) & $0.83(0.66,1.05) 12.98$ \\
\hline Li k.et al (2011) & $0.80(0.51,1.26) 9.21$ \\
\hline Takata Y.et al (2013) & $0.79(0.59,1.05) 12.06$ \\
\hline Muka T.et al (2017) & $0.80(0.57,1.13) 11.00$ \\
\hline Subtotal $(\mathrm{I}-$ squared $=0.0 \%, \mathrm{p}=0.994)$ & $0.81(0.70,0.94) 45.25$ \\
\hline \multicolumn{2}{|l|}{$>300 \mathrm{mg} / \mathrm{d}$} \\
\hline Mahabir S.et al (2008) & $0.47(0.36,0.61) 12.43$ \\
\hline Mahabir S.et al (2010) & $1.14(1.01,1.28) 14.54$ \\
\hline \multicolumn{2}{|r|}{$0.83(0.49,1.42) 7.97$} \\
\hline \multicolumn{2}{|r|}{$0.81(0.54,1.22) 9.89$} \\
\hline \multicolumn{2}{|r|}{$0.84(0.56,1.27) 9.92$} \\
\hline \multirow{2}{*}{\multicolumn{2}{|c|}{ Subtotal $(I-$-squared $=89.3 \%, p=0.000)$}} \\
\hline & \\
\hline \multicolumn{2}{|l|}{ Overall (I-squared $=80.5 \%, p=0.000)$} \\
\hline NOTE: Weights are from random effects analysi & \\
\hline $\begin{array}{l}1 \\
2\end{array}$ & \\
\hline
\end{tabular}

Figure 3. Subgroup analyses of relationship between magnesium intake and risk of lung cancer by mean dose of magnesium. 
Table 2. Newcastle-Ottawa Scale (NOS) Quality Assessment Table

\begin{tabular}{|c|c|c|c|c|c|}
\hline Author & Study & Selection & Comparability & Outcome & Score \\
\hline Takata Y. et al., 2013 & Cohort & $\star * \star$ & ** & $\star * *$ & 8 \\
\hline Muka T. et al., 2017 & Cohort & ** & * & ** & 5 \\
\hline Li K. et al., 2011 & Cohort & $* \star *$ & * & $\star \star * *$ & 7 \\
\hline Mahabir S. et al., 2010 & Case-control & $* \star \star$ & ** & 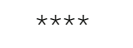 & 9 \\
\hline Mahabir S. et al., 2008 & Case-control & 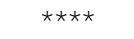 & $\star \star$ & 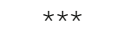 & 9 \\
\hline
\end{tabular}

Note: A star was used for assessing study quality. A study was awarded a maximum of one star for each numbered item within the selection and exposure categories. A maximum of two stars were awarded for comparability.

comparability, and outcome. Each category contains a number of questions, and based on this method, each study could ultimately have a total score of 9 points. A high score of 6 was considered as high-quality, and scores of less than or equal 6 was considered a low-quality study. The results of the NOS assessments are shown in Table 2.

\section{Publication bias}

The results of the inverse funnel diagram were almost asymmetric; however appropriate statistical tests should be used to evaluate this. As the Egger's test was significant (P-value $=0.005)$, we used the trim and fill statistical method to modify the results. The results obtained from the trim and fill test were similar to those from the metaanalysis.

\section{Discussion}

This meta-analysis examined the relationship between magnesium intake and the risk of developing lung cancer. The meta-analysis of five studies yielded a pooled RR that was not statistically significant $(\mathrm{RR}=0.72,95 \% \mathrm{CI}=0.52$ to $1.19 ; p=0.254)$. We performed a sensitivity analysis to determine the impact of each study on the final outcome, with a summary range of RR estimation of 0.78-0.97. In other words, the final result was less sensitive when entering results of each study, with the meta-analysis model conferring a higher degree of stability.

We evaluated the relationship between magnesium intake and lung cancer risk through a meta-analysis and found that an intake of dietary magnesium of less than $300 \mathrm{mg} / \mathrm{d}$ significantly reduced lung cancer risk. The potential mechanisms by which magnesium could protect against lung cancer includes its roles in maintaining genetic stability [17] regulating cell proliferation [19], protection against inflammation [20, 21] and maintenance of lung function [22].

The intake of magnesium is marginal in most industrialized countries. Recommended daily dietary allowances (RDA) of magnesium in the United States of America have increased recently to $320 \mathrm{mg}$ per day for adult women and to $420 \mathrm{mg}$ per day for adult men [31]. In Germany, Switzerland, and Austria, these values are $300 \mathrm{mg} /$ day for women and $350 \mathrm{mg} /$ day for men aged 25 years and older [17]. However, the actual magnesium intake is likely to be $67-77 \%$ of RDA in West Germany, England, and some regions of North America. Even lower magnesium intakes of barely $50 \%$ RDA were observed in some parts of Canada and Japan [17].

A case-control study of lung cancer by Mahabir et al. reports that magnesium from food sources in the typical US diet offers protection against lung cancer. They found that increasing dietary intake of magnesium was associated with a reduced lung cancer risk ranging from $17 \%$ to $53 \%$ [30]. In contrast, Takata et al. [32] suggest that the association between dietary magnesium intake and lung cancer risk (after adjustment for calcium intake) was similar to the findings reported in a cohort study by Li et al. in Germany [36]. The study by Takata et al. reported that intakes of calcium, phosphorus, and the calcium-to-magnesium (Ca:Mg) ratio were inversely associated with lung cancer risk. Our meta-analysis identified two studies that separately examined the effect of magnesium consumption on lung cancer in men; by combining the results of these two studies, the pooled RR with $95 \%$ confident interval was not statistically significant $(\mathrm{RR}=0.92,95 \% \mathrm{CI}=0.77$ to $1.11 ; p=0.382$. The effect size pooled by using a randomeffects model suggested significant heterogeneity $\left(\mathrm{I}^{2}=\right.$ $88.7 \%, \mathrm{p}<0.001)[5,30]$.

A case-control study by Mahabir et al. reported that in the low dietary $\mathrm{Mg}+$ suboptimal DNA repair capacity (DRC) group, the effects on lung cancer were more pronounced in men ( $200 \%$ increase lung cancer risk) than in women ( $\sim 80 \%$ increase lung cancer risk) [30]. A later study by the same group reported that total $\mathrm{Mg}$ intake increased the risk (Ptrend $<0.05$ ) in men and current smokers [6]. We were unable to identify a statistically significant effect of magnesium consumption on lung cancer in men when we combined the results of these two studies $[6,30]$.

There are other observational human studies on the relationship between magnesium intake and cancer risk. Some studies were inclusive of both supplemental magnesium and dietary magnesium and others either separated these or considered only dietary magnesium. Inclusion of supplementary magnesium can confound the interpretation of 
study results because the content, intake duration, and dosage of the supplementary magnesium are highly variable; in addition, the use of supplementary magnesium can influence the estimated average daily requirement $[33,34]$.

Our study has several limitations. First, there is significant heterogeneity in the limited number of published studies. Although a random effects model was used in the present meta-analysis in consideration of this, it is possible that the reliability was affected by the relatively high level of heterogeneity $(75.8 \%)$ we observed. Second, there is variation in studies we analyzed. The quality, study methods, subjects, follow-up periods and adjustment factors of each study were variable. Third, selection bias and recall bias might is possible as this meta-analysis used observational studies. Fourth, magnesium intake from drinking water was not considered. Lastly, not all studies included in this meta-analysis provided consistent information about cigarette smoking habits, so we could not undertake a subgroup meta-analysis to examine the relationship between smoking and magnesium intake on lung cancer risk. There are several compounds in cigarettes which can react with electrophilic moieties and form specific DNA adducts. Errors during the repair of the adducts by mutations in the TP53 tumor suppressor gene are important causes of smokingassociated lung cancer [35].

\section{Conclusion}

In summary, our meta-analysis shows that magnesium intake of less than $300 \mathrm{mg} / \mathrm{d}$ significantly reduced the risk of cancer lung cancer, suggesting that dietary magnesium has a protective effect on lung cancer. The importance of an adequate dietary magnesium intake in the general population should be stressed. Sufficient intake of fruits and vegetables is strongly recommended as these contain sufficient amounts of magnesium as well as other minerals and vitamins that have no adverse effects. Further randomized controlled trials on magnesium and the risk of lung cancer are recommended.

\section{References}

1. Kilgoz H.O., Bender G., \& Scandura J.M., et al. (2016) KRAS and the reality of personalized medicine in non-small cell lung cancer. Mol. Med. 22, 380-387.

2. Liu Y., Zhang J., \& Li L., et al. (2016) Genomic heterogeneity of multiple synchronous lung cancer. Nat. Commun. 7, 13200.

3. Siegel R.L., Miller K.D., \& Jemal A. (2019) Cancer statistics. CA Cancer J. Clin. 69, 7-34.

4. Ohno M.A. (2006) Genome-wide distribution of 8-oxoguanine correlates with the preferred regions for recombination and single nucleotide polymorphism in the human genome. Genome Res. 16, 567-575.
5. Mahabir S., Spitz M.R., \& Barrera S.L., et al. (2006) Dietary zinc, copper and selenium, and risk of lung cancer. Int. J. Cancer 120, 1108-1115.

6. Mahabir S., Forman M.R., \& Dong Y.Q., et al. (2010) Mineral Intake and lung cancer risk in the NIH-American Association of Retired Persons Diet and Health Study. Cancer Epidemiol. Biomarkers Prev. 19, 1976-1983.

7. Zhou W., Park S., \& Liu G., et al. (2005) Dietary iron, zinc, and calcium and the risk of lung cancer. Epidemiology 16, 772-9.

8. Lee D.-H., \& Jacobs D.R. Jr (2005) Interaction among heme iron, zinc, and supplemental vitamin $\mathrm{C}$ Intake on the risk of lung cancer: lowa women's health study. Nutr. Cancer 52, $130-137$.

9. Huang Z., Zhang Z., \& Jiang Y., et al. (2012) Targeted delivery of oligonucleotides into tumor-associated macrophages for cancer immunotherapy. J. Control. Release 158, 286-92.

10. Anghileri L.J. (2009) Magnesium, calcium and cancer. Magnes. Res. 22, 247-255.

11. Wolf F.I., Maier J.A.M., \& Nasulewicz A., et al. (2007) Magnesium and neoplasia: from carcinogenesis to tumor growth and progression or treatment. Arch. Biochem. Biophys. 458, 24-32.

12. Anastassopoulou J., \& Theophanides T. (2002) MagnesiumDNA interactions and the possible relation of magnesium to carcinogenesis. Irradiation and free radicals. Crit. Rev. Oncol. Hematol. 42, 79-91.

13. Pilchova I., Klacanova K., \& Tatarkova Z., et al. (2017) The involvement of $\mathrm{Mg}^{2+}$ in regulation of cellular and mitochondrial functions. Oxid. Med. Cell. Longev. 2017, 1-8.

14. Sahmoun A.E., \& Singh B.B. (2010) Does a higher ratio of serum calcium to magnesium increase the risk for postmenopausal breast cancer? Med Hypotheses 75, 315-8.

15. Tam M., Gómez S., González-Gross M., \& Marcos A. (2003) Possible roles of magnesium on the immune system. Eur. J. Clin. Nutr. 57, 1193-7.

16. Kasprzak K.S., \& Waalkes M.P. (1986) The role of calcium, magnesium, and zinc in carcinogenesis. Adv. Exp. Med. Biol. 206, 497-515.

17. Hartwig A. (2001) Role of magnesium in genomic stability. Mutat. Res. 475, 113-21.

18. Castiglioni S., \& Maier J.A.M. (2011) Magnesium and cancer: a dangerous liason. Magnes. Res. 24, 92-100.

19. Rubin H. (2005) Magnesium: The missing element in molecular views of cell proliferation control. Bioessays 27, 311-20.

20. King D.E., Mainous A.G., Geesey M.E., \& Woolson R.F. (2005) Dietary magnesium and C-reactive protein levels. J. Am. Coll. Nutr. 24, 166-71.

21. Song Y., Ridker P.M., \& Manson J.E., et al. (2005) Magnesium intake, C-reactive protein, and the prevalence of metabolic syndrome in middle-aged and older U.S. women. Diabetes Care 28, 1438-44.

22. Britton J., Pavord I., \& Richards K., et al. (1994) Dietary magnesium, lung function, wheezing, and airway hyperreactivity in a random adult population sample. Lancet (London, England) 344, 357-62.

23. Hans C.P., Chaudhary D.P., \& Bansal D.D. (2002) Magnesium deficiency increases oxidative stress in rats. Indian J. Exp. Biol. 40, 1275-9.

24. Shivakumar K., \& Kumar B.P. (1997) Magnesium deficiency enhances oxidative stress and collagen synthesis in vivo in the aorta of rats. Int. J. Biochem. Cell. Biol. 29, 1273-8.

25. Chen G.-C., Pang Z., \& Liu Q.-F. (2012) Magnesium intake and risk of colorectal cancer: a meta-analysis of prospective studies. Eur. J. Clin. Nutr. 66, 1182-1186.

26. Stare J., \& Maucort-Boulch D. (2016) Odds ratio, hazard ratio and relative risk. Metod. Zv. 13, 59-67. 
27. Egger M., Davey Smith G., Schneider M., \& Minder C. (1997) Bias in meta-analysis detected by a simple, graphical test. BMJ 315, 629-34.

28. Begg C.B., \& Mazumdar M. (1994) Operating characteristics of a rank correlation test for publication bias. Biometrics 50 , 1088-101.

29. Higgins J.P.T., \& Thompson S.G. (2002) Quantifying heterogeneity in a meta-analysis. Stat. Med. 21, 1539-58.

30. Mahabir S., Wei Q., \& Barrera S.L., et al. (2008) Dietary magnesium and DNA repair capacity as risk factors for lung cancer. Carcinogenesis 29, 949-56.

31. Rosique-Esteban N., Guasch-Ferré M., Hernández-Alonso P., \& Salas-Salvadó J. (2018) Dietary magnesium and cardiovascular disease: A review with emphasis in epidemiological studies. Nutrients 10.

32. Takata Y., Shu X.-O., \& Yang G., et al. (2013) Calcium intake and lung cancer risk among female nonsmokers: A report from the Shanghai Women's Health study. Cancer Epidemiol. Biomarkers Prev. 22, 50-57.

33. Shakur Y.A., Tarasuk V., Corey P., \& O'Connor D.L. (2012) A comparison of micronutrient inadequacy and risk of high micronutrient intakes among vitamin and mineral supplement users and nonusers in Canada. J. Nutr. 142, 534-40.

34. Bailey R.L., Fulgoni V.L., Keast D.R., \& Dwyer J.T. (2011) Dietary supplement use is associated with higher intakes of minerals from food sources. Am. J. Clin. Nutr. 94, 1376-81.

35. Golemis E.A., Scheet P., \& Beck T.N., et al. (2018) Molecular mechanisms of the preventable causes of cancer in the United States. Genes Dev. 32, 868-902.

36. Li K., Kaaks R., Linseisen J., \& Rohrmann S. (2011) Dietary calcium and magnesium intake in relation to cancer incidence and mortality in a German prospective cohort (EPIC-Heidelberg). Cancer Causes Control 22, 1375-1382.

37. Muka T., Kraja B., \& Ruiter R., et al. (2017) Dietary mineral intake and lung cancer risk: the Rotterdam Study. Eur. J. Nutr. 56, 1637-1646.

\section{History}

Received December 9, 2018

Accepted May 28, 2019

Published online July 15, 2019

\section{Funding}

This study was supported by Isfahan University of Medical sciences (Grant No. 295151).

\section{Conflict of interest and disclosures}

The authors declare no conflict of interest

\section{ORCID}

Golnaz Vaseghi

(iD) https://orcid.org/0000-0003-3040-6135

\section{Golnaz Vaseghi, Pharm.D, Ph.D}

Cardiovascular Research Institute

Salman-e-farsi st

Isfahan University of Medical Sciences

Isfahan, Iran

golnazvaseghi@yahoo.com 